\title{
Cowden Syndrome Diagnosed by Bilateral Breast Cancer with Lhermitte- Duclos Disease: A Case Report
}

\author{
Tamami Morisaki $^{a}$ Shinichiro Kashiwagi ${ }^{a} \quad$ Rika Kouhashia \\ Akimichi Yabumoto $^{a}$ Yuka Asano $^{a}$ Tsutomu Takashima ${ }^{a}$ \\ Kosei Hirakawa ${ }^{a, b}$ Masaichi Ohira ${ }^{a, b}$ \\ aDepartment of Breast and Endocrine Surgery, Osaka City University Graduate School of \\ Medicine, Osaka, Japan; ${ }^{b}$ Department of Gastrointestinal Surgery, Osaka City University \\ Graduate School of Medicine, Osaka, Japan
}

\section{Keywords}

Cowden syndrome · Breast cancer · Lhermitte-Duclos disease · PTEN · Thyroid tumor

\begin{abstract}
Cowden syndrome is extremely rare and is characterized by multiple hamartomas in various tissues, including the skin, mucous membranes, gastrointestinal tract, breast, thyroid, and brain, and has an increased risk of breast, thyroid, and uterine cancers. Here, we report a case of Cowden syndrome diagnosed following presentation with bilateral breast cancer and provide a discussion of the relevant literature. A 47-year-old woman with a tumor in her right breast was referred to our hospital. She was diagnosed with bilateral breast cancer upon imaging and underwent a bilateral mastectomy and sentinel lymph node biopsy. Previously, she had undergone total thyroidectomy to treat a thyroid tumor. Approximately 3 years later, she was diagnosed with Lhermitte-Duclos disease affecting her left cerebellar hemisphere. As her sister and mother had also been diagnosed with breast cancer, we suspected that she might have an inherited disease. Since $80 \%$ of individuals with Cowden syndrome have a mutation in the phosphatase and tension homolog (PTEN) gene, we did not perform any genetic testing. Instead, we used the syndrome's pathognomonic criteria and major criteria (breast cancer, thyroid tumor, and Lhermitte-Duclos disease) to diagnose our patient with Cowden syndrome. While treatment of Cowden syndrome is currently limited to strategies that can manage the symptoms, patients are at an increased risk of certain cancers and require regular screening to allow for early detection of disease.




\section{Background}

Cowden syndrome is extremely rare and is characterized by multiple hamartomas in various tissues, in particular on the skin and mucous membranes, as well as in the gastrointestinal tract, breast, thyroid, and brain [1-4]. Patients with Cowden syndrome are also at an increased risk of malignancies, especially breast cancer, thyroid cancer, and uterine cancer [1-4]. Here, we report a case of Cowden syndrome diagnosed following diagnosis of bilateral breast cancer and Lhermitte-Duclos disease and provide a discussion of the relevant literature.

\section{Case Presentation}

A 47-year-old woman with a tumor in her right breast was referred to our hospital. Both her mother and older sister had been diagnosed previously with breast cancer. She had undergone surgery twice to treat a benign thyroid tumor. During the physical examination, no mass was palpable in either breast. Ultrasonography revealed an irregular marginated hypoechoic mass measuring $15.1 \times 15.0 \times 9.4 \mathrm{~mm}$ in the 12 o'clock region of the right breast (Fig. 1a) and an irregular marginated hypoechoic mass measuring $24.8 \times 21.9 \times 10.6 \mathrm{~mm}$ in the upper inner quadrant of the left breast (Fig. 1b). Core-needle biopsies were performed at each mass site. Pathological examination of the core-needle biopsies specimen confirmed ductal carcinoma in situ (DCIS) in the right mammary gland and invasive ductal carcinoma in the left mammary gland. Computed tomography scan did not reveal lymph node metastases or distant metastases (Fig. 2a). Contrast-enhanced magnetic resonance imaging (MRI) revealed the primary tumor of the right mammary gland to be a mass $15 \mathrm{~mm}$ in size and likely to be DCIS (Fig. 2b). Contrast-enhanced MRI of the left mammary gland exhibited early phase linear enhancement (Fig. 2c). Bone scintigraphy revealed no metastasis to the bone. The preoperative diagnosis was stage 0 (cTisN0M0) right breast cancer and stage IIA (cT2N0M0, estrogen receptor [ER]-negative, progesterone receptor [PgR]-negative, and human epidermal growth factor receptor 2 [HER2]-negative, with low Ki67 expression) left breast cancer. Surgery consisted of a bilateral mastectomy and sentinel node biopsy. The final diagnosis was bilateral breast cancer (right: pTisN0M0 stage 0, DCIS) (left: pT2N0M0 stage IIA, invasive
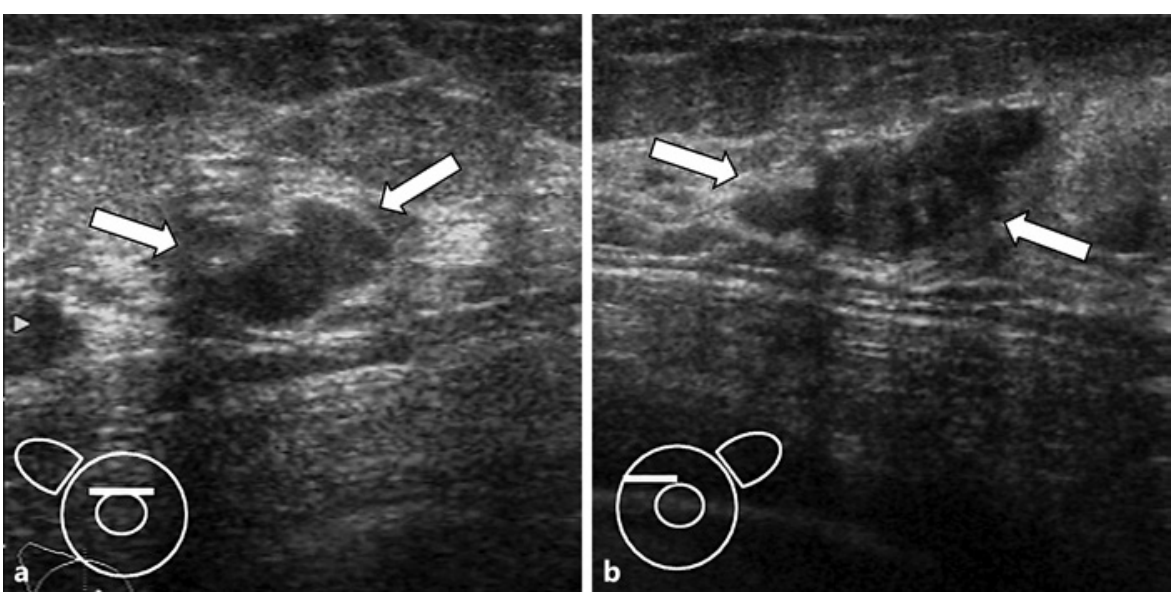

Fig. 1. Ultrasonography findings. Breast ultrasonography revealed an irregular marginated hypoechoic mass measuring $15.1 \times 15.0 \times 9.4 \mathrm{~mm}$ in the upper middle of the right breast (a) and an irregular marginated hypoechoic mass measuring $24.8 \times 21.9 \times 10.6 \mathrm{~mm}$ in the upper inner quadrant of the left breast (b). 

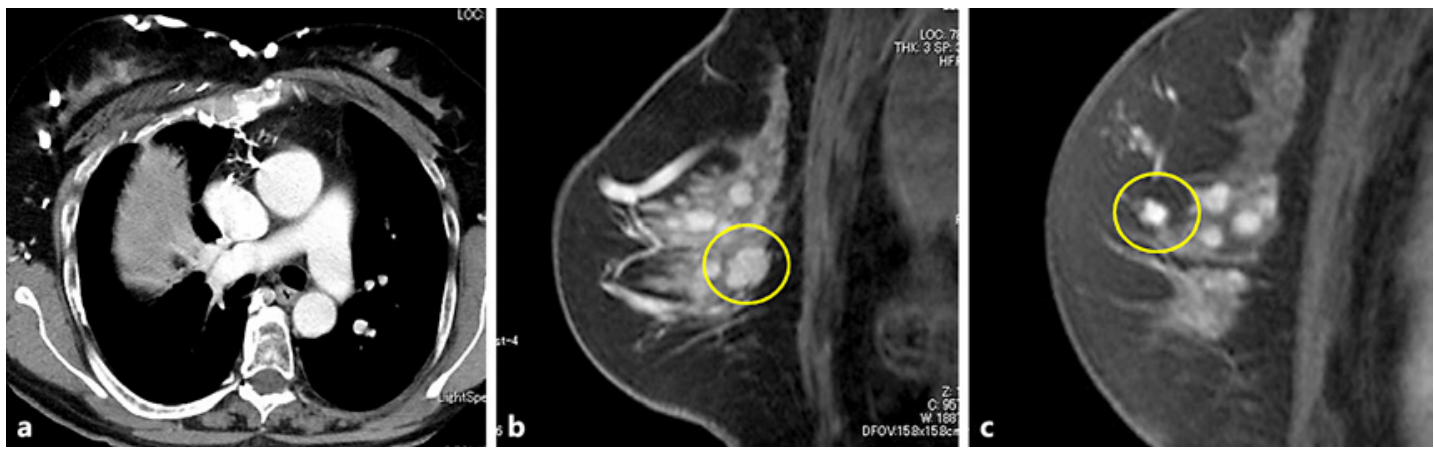

Fig. 2. Computed tomography (CT) and magnetic resonance imaging (MRI) findings of the chest. CT scan did not reveal lymph node metastases or distant metastases (a). Contrast-enhanced MRI revealed the primary tumor of the right mammary gland to be a mass $15 \mathrm{~mm}$ in size and likely to be DCIS (b). Contrast-enhanced MRI of the left mammary gland exhibited early-phase linear enhancement (c).
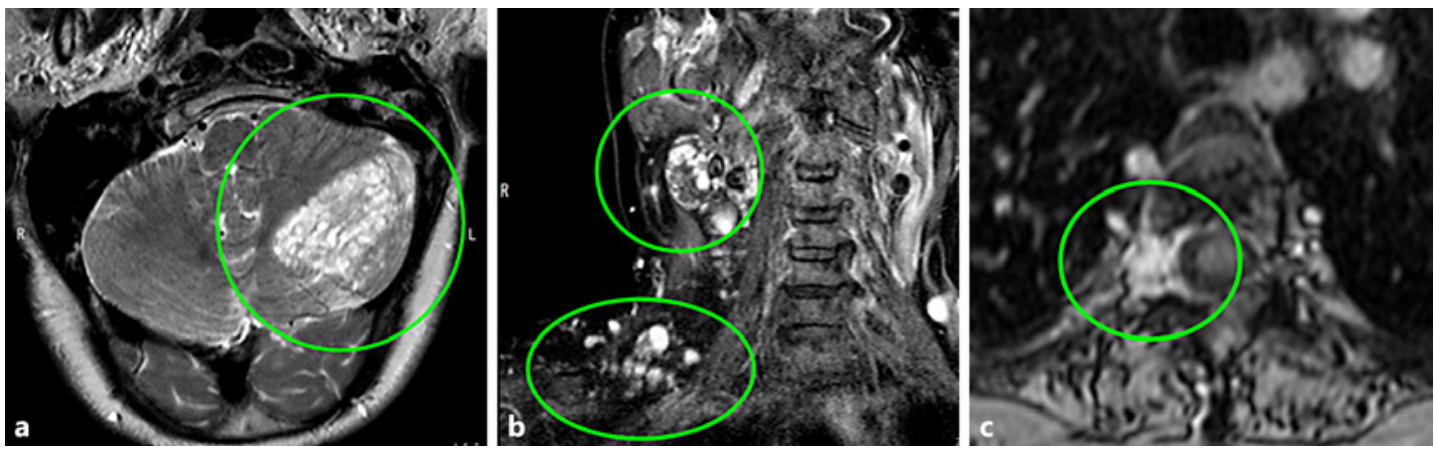

Fig. 3. Magnetic resonance imaging (MRI) findings of the brain. MRI showed alternative isointense and hyperintense bands in the left cerebellar hemisphere. a Transverse plane. b, c Coronal plane.

ductal carcinoma, nuclear grade 1, ER-negative, PgR-negative, and HER2-negative, with low Ki67 expression). Following surgery, the patient was treated with systemic adjuvant endocrine therapy (anastrozole $1 \mathrm{mg} /$ day). After 3 years of adjuvant treatment and observation, there has been no evidence of recurrence or metastasis. However, she was diagnosed with Lhermitte-Duclos disease after imaging her left cerebellar hemisphere. MRI of the brain showed alternative isointense and hyperintense bands in the left cerebellar hemisphere (Fig. 3a-c). As her sister and mother also had breast cancer, we suspected that she may have an inherited disorder.

Although phosphatase and tension homolog (PTEN) genetic testing was not performed, we could diagnose this patient as having Cowden syndrome using the pathognomonic criteria and major criteria of Cowden syndrome (breast cancer, thyroid tumor, and Lhermitte-Duclos disease).

\section{Discussion/Conclusion}

Cowden syndrome is a rare, multisystem disease that causes an increased risk of malignancies (breast, thyroid, and endometrial) as well as benign hamartomatous overgrowth of tissues (skin, colon, thyroid, etc.) [1-5]. Cowden syndrome was first described in 1 family in 
Morisaki et al.: Cowden Syndrome with Bilateral Breast Cancer and Lhermitte-Duclos Disease

1963 [6]. In the Western hemisphere, the frequency of this syndrome is 1 in every 200,000 people, but it is likely to be more frequent because some cases are overlooked [1,7]. The diagnostic criteria proposed by the International Cowden Consortium (ICC) and the National Comprehensive Cancer Network (NCCN) are widely used [8]. It has been reported that $90 \%$ of individuals that meet the criteria for ICC develop clinical symptoms by the age of 20 years [1].

This syndrome is caused by a loss-of-function germline mutation in the PTEN gene located on the long arm of chromosome 10 (10q23.31) [5, 9]. The PTEN gene is a tumor suppressor gene that encodes a lipid kinase that regulates cell proliferation and cell death by suppressing PIK3/AKT pathway signaling [2]. Changes in somatic cells are observed in cancers with PTEN mutations, such as endometrioid adenocarcinoma, glioblastoma, and prostate cancer. A family history of Cowden syndrome is confirmed in $10-50 \%$ of patients, and about $18 \%$ of patients do not have mutations in the PTEN gene [2].

This disease can cause multiple hamartomas in various tissues (skin, mucous membrane, gastrointestinal tract, breast, thyroid, and brain) $[1,10,11]$. Skin growths are seen in $90-100 \%$ of patients and are characterized by multiple papules on the face [12]. Benign breast lesions are found in $76 \%$ of Cowden syndrome patients. Breast cancer is the most commonly observed malignant tumor (30-50\%) in this population, with mostly invasive cancers. Breast cancers are often discovered at a younger age (38-46 years) than average, and men with Cowden syndrome are at a higher risk of breast cancer than women [5]. The luminal subtype is often the breast cancer subtype reported $[4,5]$. Further, $3-10 \%$ of patients with Cowden syndrome have a history of thyroid cancer, and follicular cancer is more common than papillary cancer [8]. Some patients have also been diagnosed with Lhermitte-Duclos disease, caused by a hamartoma such as a dysplastic ganglion cell tumor or a hemangioma in the cerebellum. This is a characteristic criterion of Cowden syndrome as proposed by the NCCN [8].

NCCN guidelines for genetic/familial risk assessment in breast and ovarian cancer recommend regular screening for comorbid tumors for individuals with PTEN hamartoma syndrome diagnosed by genetic testing and Cowden syndrome diagnosed clinically [8]. This case was considered to be an inherited disease based on the patient's medical and family history. Eighty percent of patients with Cowden syndrome harbor a PTEN mutation. Although we did not perform genetic testing, we could use the pathognomonic criteria and major criteria of Cowden syndrome (breast cancer, thyroid tumor, and Lhermitte-Duclos disease) to diagnose this patient [13].

While treatment of Cowden syndrome is currently limited to strategies that can manage the symptoms, patients are at an increased risk of certain cancers and require regular screening to allow for early detection of disease.

\section{Acknowledgements}

We thank Yayoi Matsukiyo and Tomomi Okawa (Department of Breast and Endocrine Surgery, Osaka City University Graduate School of Medicine) for helpful advice regarding data management.

\section{Statement of Ethics}

Written ethical approval for the publication of the present case report was obtained from the patient. 
Morisaki et al.: Cowden Syndrome with Bilateral Breast Cancer and Lhermitte-Duclos Disease

\section{Disclosure Statement}

The authors declare that they have no conflicts of interest to disclose.

\section{Funding Sources}

No funding was received for this article, and the authors have no conflicts of interest directly relevant to this report.

\section{Author Contributions}

All authors were involved in the preparation of this manuscript. T.M. collected the data and wrote the manuscript. S.K., R.K., Y.A., and T.T. performed the operation and designed the study. S.K. and K.H. summarized the data and revised the manuscript. K.H. and M.O. made substantial contributions to the study design, performed the operation, and revised the manuscript. All authors read and approved the final manuscript.

\section{References}

1 Pilarski R. Cowden syndrome: a critical review of the clinical literature. J Genet Couns. 2009 Feb;18(1):13-27.

2 Pilarski R, Stephens JA, Noss R, Fisher JL, Prior TW. Predicting PTEN mutations: an evaluation of Cowden syndrome and Bannayan-Riley-Ruvalcaba syndrome clinical features. J Med Genet. 2011 Aug;48(8):505-12.

3 Kimura F, Ueda A, Sato E, Akimoto J, Kaise H, Yamada K, et al. Hereditary breast cancer associated with Cowden syndrome-related PTEN mutation with Lhermitte-Duclos disease. Surg Case Rep. 2017 Dec;3(1):83.

4 Walsh S, Carter M, Tubridy N, McDermott EW. Lhermitte-Duclos and Cowden diseases: breast cancer as an unusual initial presentation of these overlapping conditions. BMJ Case Rep. 2011 Oct 20;2011.

5 Schrager CA, Schneider D, Gruener AC, Tsou HC, Peacocke M. Clinical and pathological features of breast disease in Cowden's syndrome: an underrecognized syndrome with an increased risk of breast cancer. Hum Pathol. 1998 Jan;29(1):47-53.

6 Lloyd KM 2nd, Dennis M. Cowden's disease. A possible new symptom complex with multiple system involvement. Ann Intern Med. 1963 Jan;58:136-42.

7 Pilarski R. PTEN hamartoma tumor syndrome: A clinical overview. Cancers (Basel). 2019 Jun 18;11(6):pii: E844.

8 National Comprehensive Cancer Network. Cowden syndrome clinical practice guidelines in oncology. https: //www.nccn.org/professionals/physician_gls/default.aspx

9 Liaw D, Marsh DJ, Li J, Dahia PL, Wang SI, Zheng Z, et al. Germline mutations of the PTEN gene in Cowden disease, an inherited breast and thyroid cancer syndrome. Nat Genet. 1997 May;16(1):64-7.

10 Bubien V, Bonnet F, Brouste V, Hoppe S, Barouk-Simonet E, David A, et al. High cumulative risks of cancer in patients with PTEN hamartoma tumour syndrome. J Med Genet. 2013 Apr;50 (4):255-63.

11 Heindl M, Händel N, Ngeow J, Kionke J, Wittekind C, Kamprad M, et al. Autoimmunity, intestinal lymphoid hyperplasia, and defects in mucosal B-cell homeostasis in patients with PTEN hamartoma tumor syndrome. Gastroenterology. 2012 May;142(5):1093-e6.

12 Hildenbrand C, Burgdorf WH, Lautenschlager S. Cowden syndrome-diagnostic skin signs. Dermatology (Basel). 2001;202(4):362-6.

13 Eng C. Will the real Cowden syndrome please stand up: revised diagnostic criteria. J Med Genet. 2000 Nov; 37(11):828-30. 